Review

\title{
Photoacoustic-Based Multimodal Nanoprobes: from Constructing to Biological Applications
}

\author{
Duyang Gao, Zhen Yuan ${ }^{\bowtie}$ \\ Bioimaging Core, Faculty of Health Sciences, University of Macau, Macau SAR, China \\ $\triangle$ Corresponding author: Zhen Yuan, zhenyuan@umac.mo \\ (c) Ivyspring International Publisher. This is an open access article distributed under the terms of the Creative Commons Attribution (CC BY-NC) license \\ (https:// creativecommons.org/licenses/by-nc/4.0/). See http://ivyspring.com/terms for full terms and conditions.
}

Received: 2016.12.13; Accepted: 2017.02.05; Published: 2017.02.25

\begin{abstract}
Multimodal nanoprobes have attracted intensive attentions since they can integrate various imaging modalities to obtain complementary merits of single modality. Meanwhile, recent interest in laser-induced photoacoustic imaging is rapidly growing due to its unique advantages in visualizing tissue structure and function with high spatial resolution and satisfactory imaging depth. In this review, we summarize multimodal nanoprobes involving photoacoustic imaging. In particular, we focus on the method to construct multimodal nanoprobes. We have divided the synthetic methods into two types. First, we call it "one for all" concept, which involves intrinsic properties of the element in a single particle. Second, "all in one" concept, which means integrating different functional blocks in one particle. Then, we simply introduce the applications of the multifunctional nanoprobes for in vivo imaging and imaging-guided tumor therapy. At last, we discuss the advantages and disadvantages of the present methods to construct the multimodal nanoprobes and share our viewpoints in this area.
\end{abstract}

Key words: multimodal nanoprobes, synthesis methods, photoacoustic imaging, biomedical imaging.

\section{Introduction}

Various kinds of biomedical imaging modalities have been developed in the past decades, such as optical imaging, ultrasound (US) imaging, computed tomography (CT), and magnetic resonance imaging (MRI) [1-4]. Of all the imaging methods, optical imaging is a modality that offers unique advantages of high sensitivity, inexpensive and noninvasive, although it has limited imaging depth due to the effect of optical scattering. In addition, US imaging is extensively adopted in the hospital because of their easy handling, safety and low-cost. However, it is hard to use US to generate good reproducible data and high sensitivity results [5-6]. CT is a mature technique that uses $\mathrm{x}$-ray as the laser sources to generate three-dimensional structural images of biological tissues with high resolution. Meanwhile, it has contrast problem for imaging the soft tissue and the risks of radiation also exists during the CT scan [7-8]. MRI instrument is another routinely used method in clinical practice, which can detect the anatomy or functional information of biological tissues with high resolution and excellent imaging depth [9, 10]. However, MRI also exhibits the disadvantages of time-consuming and high cost, which makes it hard to get real-time information for disease diagnosis. As described above, we can conclude that all of the imaging modalities have their own advantages and intrinsic drawbacks (Table 1). Therefore, it is of great importance to combine different imaging modalities together to obtain more tissue information. Consequently, it is essential to take advantages of the complementary information from multiple imaging techniques to capture more tissue information that may be missed by a single one. It is also anticipated that multimodality imaging approaches can detect the diseased tissues with high sensitivity, high resolution, high contrast, and satisfactory imaging depth. 
Table 1. Advantages and Disadvantages of current imaging modalities for molecular imaging

\begin{tabular}{|c|c|c|}
\hline Imaging modality & Advantages & Disadvantages \\
\hline Optical Imaging & High-throughput screening; High sensitivity; Multicolor imaging & Low depth penetration; Low spatial resolution \\
\hline Magnetic Resonance Imaging & Deep penetrating; High spatial resolution & High Costs; Long imaging time; Low sensitivity \\
\hline Ultrasound Imaging & Real-time imaging; Low costs & Operator dependency; Low resolution \\
\hline Positron-Emission Tomography Imaging & $\begin{array}{l}\text { Whole-body scanning; High sensitivity; Unlimited depth } \\
\text { penetration }\end{array}$ & High costs; Radiation risk \\
\hline $\begin{array}{l}\text { Single-Photon-Emission Computed } \\
\text { Tomography Imaging }\end{array}$ & High sensitivity; No tissue penetrating limit & Radiation risk \\
\hline Computed Tomography Imaging & High spatial resolution; No tissue penetrating limit & Radiation risk; Poor soft-tissue contrast \\
\hline
\end{tabular}

Photoacoustic (PA) imaging based on PA effect, is growing rapidly, which paves a new avenue to link a complex biological system at multiple length scales [11]. In PA imaging, photons are absorbed by the biological tissue that can produce a very small change of temperature [12]. The little temperature variation of the biological tissue will cause thermo-elastic expansion, which can further generate acoustic waves that can be detected by ultrasound transducers around the tissue surface. Then the functional and anatomical information of biological tissue can be recovered based on measured acoustic pressures. As illustrated in Figure 1, PA imaging that combines the advantages of optical imaging and ultrasonic imaging, possesses the deep penetration depth and excellent spatial resolution due to the low scattering of acoustics, which is nearly three orders smaller than that of light in biological tissue. Simultaneously, the imaging contrast of PA imaging is also very high due to the significantly increased vascularity in diseased tissues. However, the sensitivity of PA imaging to detect the diseased tissues are dominated by the endogenous contrast, which can't distinguish well between normal and abnormal tissues, especially at the early stage of the diseases [9]. Therefore, it is essential to design exogenous contrast agents to improve the sensitivity of PA imaging. To date, various categories of contrast agents have been developed and used for PA molecular imaging. Among all the imaging agents, nanoprobes have received considerable attentions because of their unique magnetic, optical and electronic properties [13].

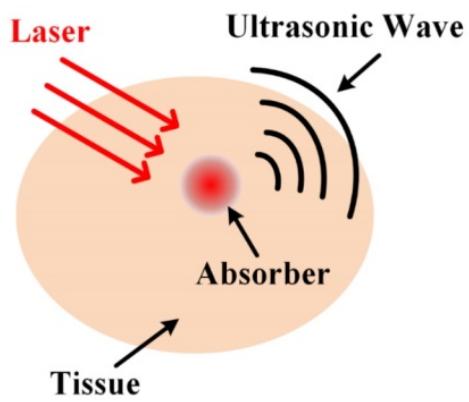

Figure 1. Schematic explain of laser-induced photoacoustic effect in tissues.
Multimodal nanoprobe is a concept of integrating more than one imaging modality agents to obtain complementary functions to improve the imaging sensitivity and specificity [14]. One of the earliest multimodal nanoprobes is introduced by Lee Josephson et al., in which they have combined near-infrared fluorescence imaging and MRI together to quantify the tumor margins. In particular, the multimodal contrast agent is composed of commercial near-infrared dye that is responsible for optical imaging and iron oxide nanoparticles that supply the function of MRI. By integrating the two modalities together, the multimodal nanoprobe can be used for intra-operative imaging since optical imaging is a real-time imaging technique, which can cover the shortage of MRI. The significant advantage of this multimodal nanoprobe is that it provides a method to visualize brain tumor preoperative and intraoperative [15]. In addition to combining fluorescence imaging and MRI, other imaging modalities have also been integrated for the purpose of multimodality imaging based on constructed multimodal nanoprobes, such as fluorescence imaging/PA/MRI, fluorescence imaging/CT/MRI, and so on [16-19]. Interestingly, several reviews on the development and application of the multimodal nanoprobes have been performed from different perspectives [20-23]. By contrast, for the present review we mainly focus on the introduction on how to design PA-based multimodal nanoprobes. In particular, this work aims to summarize the recent methods that can be used to construct PA-based multimodal nanoprobes.

\section{Construction of PA-based multimodal nanoprobes}

According to the structure of the multimodal nanoprobes, we mainly separate the multifunctional agents into two types. The first type are "one for all" nanoprobes, which involved in preparing intrinsic multimodal properties of the element into one single particle, such as gold-based nanoparticles [24-26], copper-based nanoparticles [27-28], and tungsten-based nanoparticles and so on [29]. The second type are "all in one" nanoprobes. Preparation 
of this category of multimodal nanoprobes needs to combine different imaging modality components into one single particle. There are mainly three ways to prepare "all in one" multimodal nanoprobes: i) doping different components into core material to realize multifunction; ii) encapsulating or loading different imaging components into one carrier; iii) conjugating different kinds of functional constituents with covalent or non-covalent linkage.

\section{One for all}

One of the most popular "one for all" multimodal nanoprobes is two-dimensional nanomaterials. Two-dimensional nanomaterials have been successfully applied in the areas of energy storage and lithium ion battery. The synthesize of two-dimensional nanomaterials has attracted extensive attention in the biomedical imaging field, especially after the introduction of A. Geim and K. Novoselov's work in graphene [30-35]. Besides graphene, transition-metal-based two dimensional nanomaterials have also been introduced into biological area and become very popular in very recent years [36-38]. For example, Liu Zhuang et al. have developed a dual-modal tungsten sulfide nanosheets for CT and PA imaging [29]. The nanosheets are prepared through a "top-down" chemical method, in which N-bultyllithium is used to exfoliate bulk tungsten sulfide. To realize biocompatibility and water-solubility, polyethylene glycol is conjugated on the surface of the nanosheets. The as-prepared nanosheets possess a strong near-infrared absorption with a diameter of 50-100 nm and a thickness of about $1.6 \mathrm{~nm}$. The near-infrared absorption make the nanosheets good candidates as contrast agents for PA imaging. In addition, the synthesized nanoprobe has been tested to measure whether it can be used for CT imaging. As tungsten is an element with atomic number 74, it can absorb the energy of X-ray. The results demonstrate the nanosheets can be used to enhance the CT imaging signal. The enhancement also depends on the concentration of the nanosheets. The multimodal nanoprobe is applied for in vivo biomedical imaging. In summary, Liu Zhuang's group has developed a novel multimodal nanoprobe for CT imaging and PA imaging based on the tungsten. Besides tungsten-based two-dimensional nanomaterials, cobalt selenides nanoplates are also developed and served as multimodal nanoprobe [36]. As shown in Figure 2, Poly-acrylic-acid-modified cobalt selenides nanoplates exhibit their strong near-infrared absorption and remarkable magnetic properties. The findings prove that the cobalt selenides nanoplates can be used as dual-modal PA imaging/MRI contrast agent.

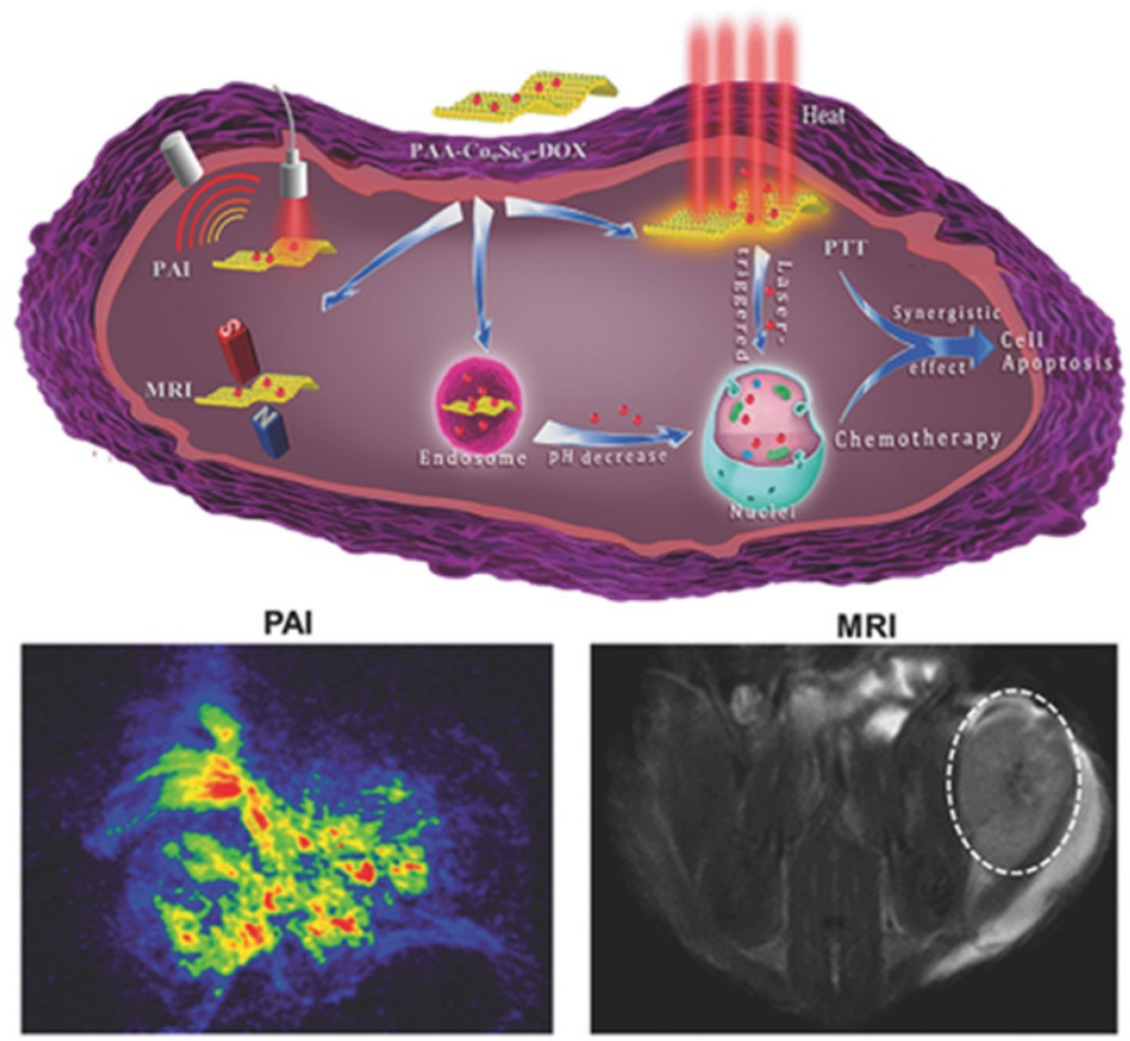

Figure 2. Schematic illustration of the designed PAA-Co9Ses -DOX theranostic platform. Reproduced with permission from ref. 36 . Copyright @ 2015 WILEY-VCH Verlag GmbH \& Co. KGaA, Weinheim. 
Another kind of "one for all" multimodal nanoprobe is copper-based nanoparticles. Copper-based nanoparticles is widely used for PA imaging because of their tunable strong near-infrared absorption. Various kinds of copper-based nanoparticles have been prepared such as CuSe nanoparticles, CuS nanoparticles, and $\mathrm{CuTe}$ nanoparticles and so on [39-42]. Very recently, CuS nanoparticle as a multimodal nanoprobe has been discovered by Mou Juan in Chen Hangrong's group [27]. Different from the above "top to down" method, CuS is prepared through "bottom to up" method. "Bottom to up" means the nanoparticles form from small molecular to nanoscale, which is also a popular chemical method to synthesize nanoparticles. The same with the fist method, surface modification process with the usage of biomimetic phospholipid is carried out to make the nanoprobe water-dispersible. In addition to increase the dispersity in the physiological environment, the modification also prolong the circulation time of the nanoprobe in the blood. As-synthesized CuS nanoprobe has a characterized absorption in the range of the second near-infrared window centered at about $1160 \mathrm{~nm}$, in which photons can penetrate much deeper in the biological tissues. The absorption of the CuS nanoprobe make it a good contrast agent for PA imaging and infrared thermal imaging. Furthermore, Chen Hangrong's group applies the as-synthesized $\mathrm{CuS}$ nanoprobe not only for PA imaging and infrared thermal imaging, but also for MRI. That was the first work for CuS nanoprobe to be used as MRI contrast agent. The MRI ability of the CuS nanoprobe is likely ascribed to the unpaired electrons of the copper. The novel multimodal nanoprobe is successfully used for in vivo tumor imaging. In the article, CuS nanoprobe has proved to be a good "one for all" PA/MRI multimodal nanoprobe. Interestingly, another group in Toyo University has revealed that $\mathrm{Cu}_{2} \mathrm{~S}$ is an effective x-ray probe [28]. They have prepared the $\mathrm{Cu}_{2} \mathrm{~S}$ nanostructure via hot injection, which is a promising method to obtain nanostructures with good crystallinity and excellent mono-dispersity. Polyethylene glycol is introduced as an excellent biocompatible polymer to prevent the aggregation of the nanoprobe when it dispersed in physiological environment. An absorption peak in the range of near-infrared region is also observed, which is in consistent of the former article. Consequently, the developed probe can also be applied for PA imaging. Since copper has a higher attenuation coefficient than the component in human body, Prof. D. Sakthi Kumar's also validates for the first time that $\mathrm{Cu}_{2} \mathrm{~S}$ nanoprobe is suitable for enhancing $\mathrm{x}$-ray imaging signal. It is also the first time for $\mathrm{Cu}_{2} \mathrm{~S}$ used as $\mathrm{x}$-ray contrast agent.

In addition to two-dimensional and copper-based multicomponent multimodal nanoprobes, single component multimodal nanoprobes have also established. For instance, gold-based multimodal nanoprobes such as gold nanoshell, gold cube, and gold nanorods are widely used for PA imaging and x-ray [24-26]. Recently, carbon dots has been designed for multimodal imaging as single component contrast agent. Carbon dots with red-emitting is constructed by Wang Pengfei's group [43], in which polythiophene phenylpropionic acid is used as the carbon source to produce the multimodal nanoprobe (Figure 3). The prepared carbon dots exhibit a broad absorbance ranging from visible to near-infrared region and a red-emitting with a peak centered at about $640 \mathrm{~nm}$. For in vivo biological applications, the stability of the carbon dots dispersed in PBS and FBS are measured. The results show the nanoprobe is dispersed well in these solutions. Because of their strong absorption and red emission, the carbon dots are applied for in vivo tumor PA imaging and fluorescence imaging. In this paper, carbon dots are proved to be excellent candidate for in vivo multimodal imaging.

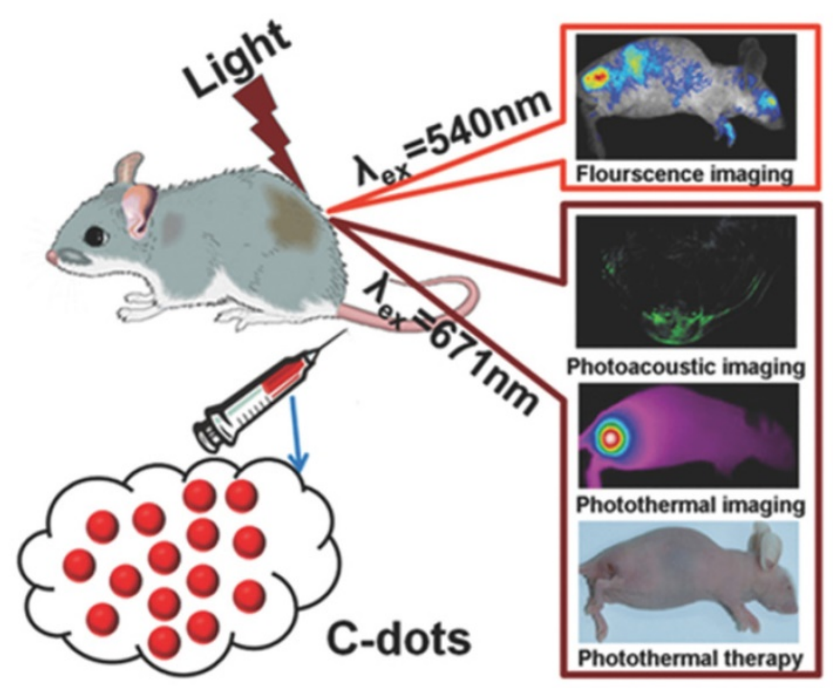

Figure 3. Schematic illustration for the design of C-dots as a theranostic nanoprobe. Reproduced with permission from ref. 43. Copyright (C) 2015 WILEY-VCH Verlag GmbH \& Co. KGaA, Weinheim.

\section{All in one}

Prior to the introduction of the methods on how to integrate different modality components together, characteristic of the common functional materials will be summarized briefly. Paramagnetic metal ions (gadolinium ions, manganese ions) and their derivations are often involved in MRI. Heavy atoms 
containing nanomaterials such as gold, iodine are always developed to construct CT contrast agents. Present nanomaterials for PA imaging often have adequate optical absorption in common that can absorb energy from the excited laser source and convert the energy into heat. Elements with radiation are participated in the positron emission tomography whereas small molecular dyes and quantum dots are often used as fluorescence imaging materials. In this section, the combination method to construct PA-based multimodal nanoprobes are organized into three small parts for the introduction of each category of all-in-one agents.

\section{Doping different components into core material}

Doping functional components into core nanomaterial to realize multimodal is a promising method to construct multimodal nanoprobes. The method is always very simple, which involves in one-pot synthesis. The process is very similar to prepare "one for all" multimodal nanoprobes. The difference between doping process and "one for all" process is the source of the multimodal imaging ability. For example, tungsten sulfide nanoprobe mentioned above is used as the contrast agent for CT and PA imaging. The source of the multimodal imaging ability is mainly due to tungsten, whereas sulfur which does not supply imaging modality is just working as an element to help tungsten to form the nanoprobe. Therefore, tungsten sulfide is classified as "one for all" multimodal nanoprobes. In contrast, doping method, we need introduce another functional component into a core material which has imaging ability of its own as well, in which both dopants and host are used as imaging components.
Inorganic ions doped into inorganic nanoparticles as a sub-branch of the doping method [44-45]. A multimodal nanoprobe for PA imaging and MRI is constructed through this method (Figure 4) [46]. To construct the multimodal nanoprobe, copper sulfide and paramagnetic ions are chosen for different imaging modalities sources. Copper sulfide nanoparticle is chosen as the core shell since its local surface plasmonic resonance in the near-infrared region. Transition metal nickel ion is chosen as the doping element because of its magnetic properties. The doped magnetic element supplies MRI ability. The multimodal nanoprobe is prepared through one-pot reaction under high temperature. The as-prepared nickel-doped copper sulfide multimodal nanoprobe has retained the absorption of the copper sulfide. The absorption peak is centered at about $1064 \mathrm{~nm}$, which is located in the region of the second biological window for PA imaging. T1 relaxivity of the doped nanoprobe is also measured. Interestingly, T1 relaxivity of the multimodal nanoprobe has been dramatically enhanced. We ascribe the enhancement of $\mathrm{T} 1$ relaxivity to the geometrical confinement of the nanostructure. T1 relaxivity is of great importance for MRI contrast agents. After ligands exchange with PEGylated multidentate polymer, the water-dispersible multimodal nanoprobe is applied for in vivo PA and magnetic resonance lymph node imaging. As we can see in this example, copper sulfide is the source of PA imaging ability and doped nickel is the source of MRI ability. The copper sulfide and nickel ions react with each other and form a whole uniform nanostructure. The properties of the components influence each other. Therefore, this multimodal nanoprobe is classified as "all in one".

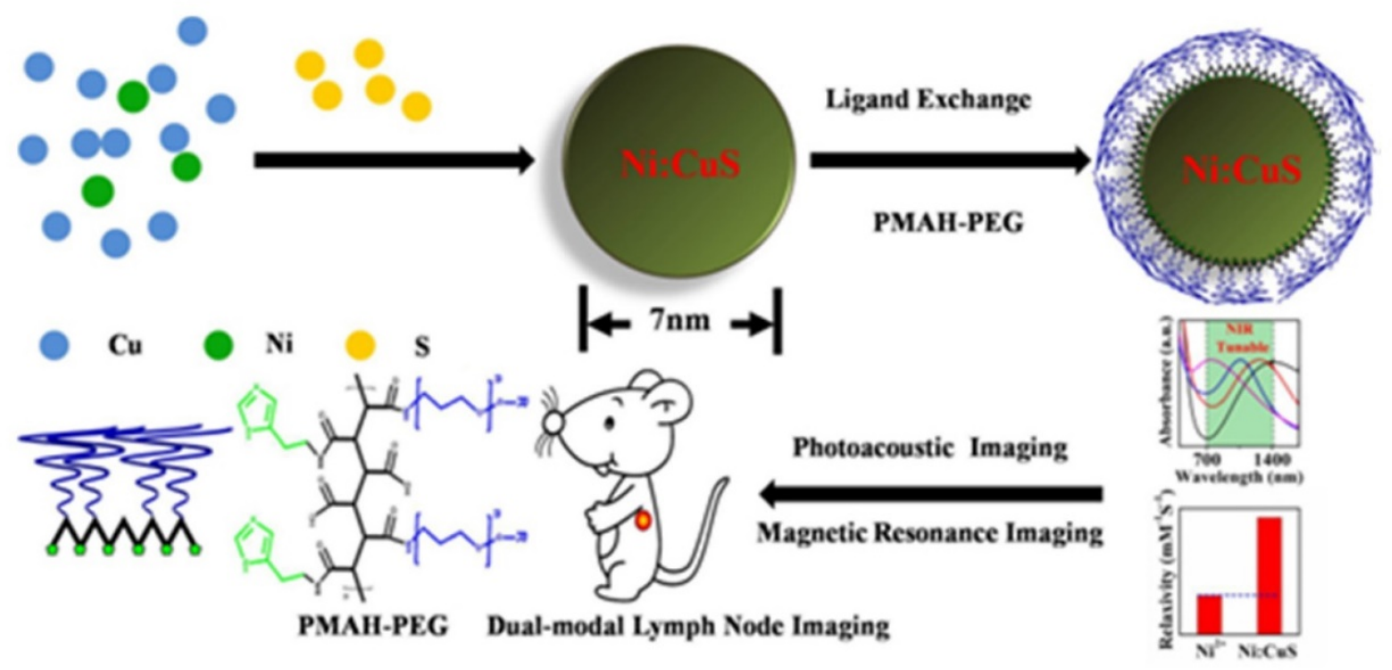

Figure 4. Schematic illustration of the designed chelator-free Ni-doped CuS NPs for in lymph node imaging. Reproduced with permission from ref. 46. Copyright (C) 2015, Royal Society of Chemistry. 

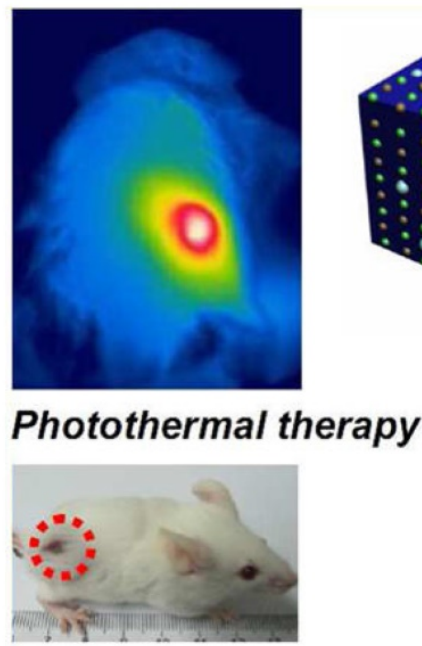

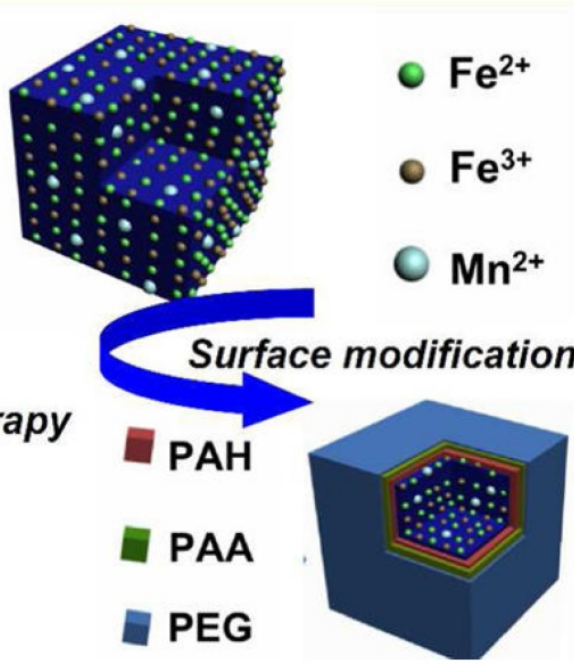

Figure 5. Scheme illustration of the constructed PB:Mn-PEG multimodal nanoprobe. Reproduced with permission from ref. 47. Copyright @ 2015, American Chemical Society.

Another type of doping is inorganic ions doping into organic nanoparticles [42]. Paramagnetic manganese ions which provide MRI ability is doped into Prussian blue nanocubes (Figure 5). Prussian blue is a clinical agent that is approved by Food and Drug Administration in the United States of America for the treatment of radioactive exposure. Recently, Prussian blue nanocubes have been designed for photothermal therapy since they have a characteristic absorption peak at the wavelength of 700nm. Liu Zhuang's group have constructed a multimodal nanoprobe for PA imaging and MRI based on the Prussian blue nanocube. Manganese ions doped Prussian blue nanocubes are prepared under mild reaction condition. The absorption and magnetic properties of the cubes with different ratios of manganese are also inspected, in which the optical and T1 relaxivity properties of the doped nanocubes exhibit concentration-dependent. The absorption peak is red-shift with the addition of manganese while T1 relaxivity is increased with increment of manganese doping. The manganese doped Prussian blue nanocubes show their great capacity for PA imaging and MRI. In conclusion, doping method is an effective method to design multimodal nanoprobes.

\section{Loading or encapsulating different imaging components into one carrier}

Loading or encapsulating different imaging components into one carrier means that there is no chemical interaction between the components. The carrier has no imaging ability. Imaging components themselves can be regarded as carriers to load or encapsulate the components. Polymers such as polysaccharide, polypeptide and silicon are also frequently used as carriers to load or encapsulate the components.

A lot of multimodal nanoprobes are established through this method [48-56]. For example, Zhao et al. have developed a multifunctional nanoprobe that integrate molybdenum sulfide and iron oxide (Figure 6) [57]. Molybdenum sulfide is a two-dimensional nanomaterials with highly near-infrared photothermal transformation efficiency. This property makes it a good candidate for PA imaging. Iron oxide nanoparticles is often used as MRI contrast agents. Zhao Yuliang et.al. integrates the molybdenum sulfide and iron oxide through locking effect of polyethylene glycol. There is only physical interaction between the two components. As the two dimensional nanomaterial possesses large surface area, molybdenum sulfide is not only used as PA imaging contrast agent, but also an excellent carrier to load iron oxide nanoparticles. The as-prepared nanoprobe is constructed through two steps. It exhibits both PA imaging and MRI ability. It is further applied for dual-modal in vivo imaging. In addition, Chen et al. have developed a multimodal nanoprobe for PA imaging and fluorescence imaging with the usage of the same method. They used graphene oxide as the carrier and source of PA imaging modality, and sinoporphyrin sodium is loading on the surface of the graphene oxide to supply fluorescence. Another PA imaging and fluorescence imaging multimodal nanoprobe has also been constructed by the group using this method. Instead of sinoporphyrin sodium, they used cy5.5 as the source of fluorescence (Figure $7)$. Graphene oxide play the same role as mentioned above $[58,59]$. 


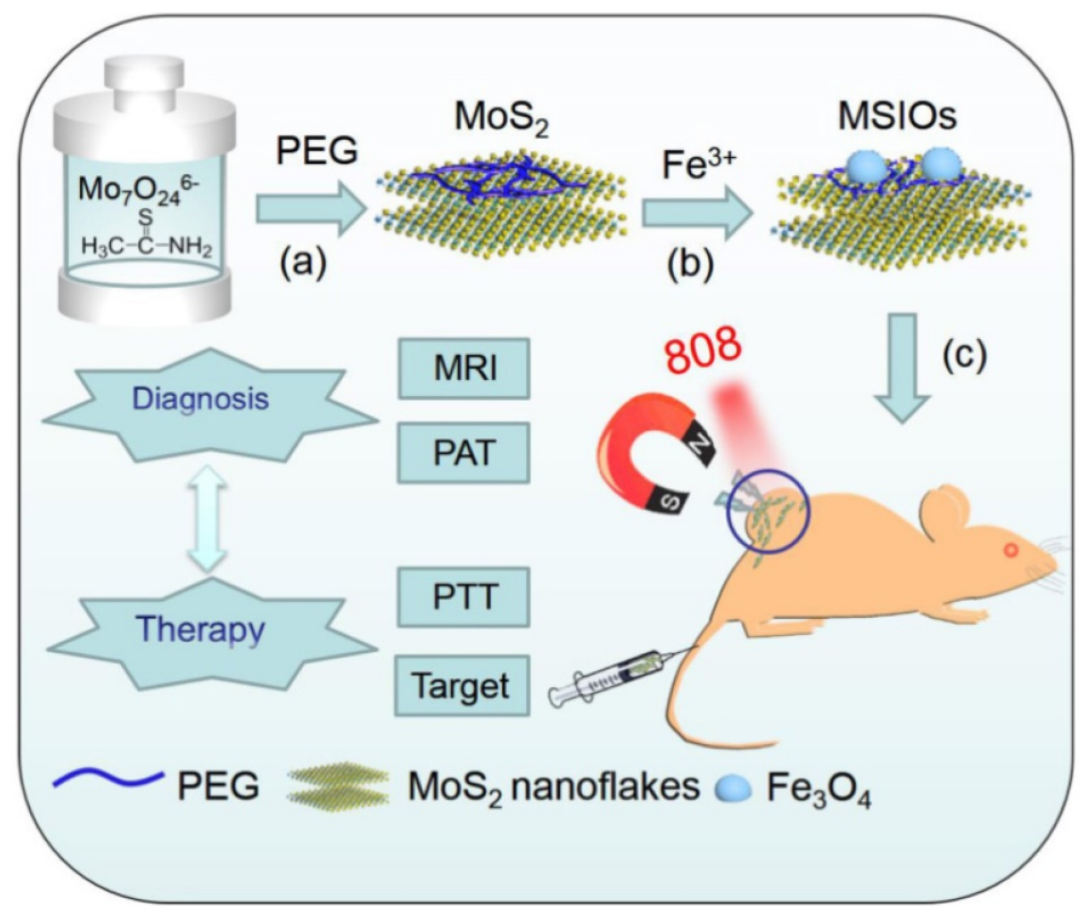

Figure 6. Schematic illustration of synthesis route of MSIOs for dual-modal magnetic resonance and photoacoustic image-guided magnetic targeting and photothermal ablation of cancer. Reproduced from reference 57. (an open-access journal printed by Ivyspring International Publisher, free to use).

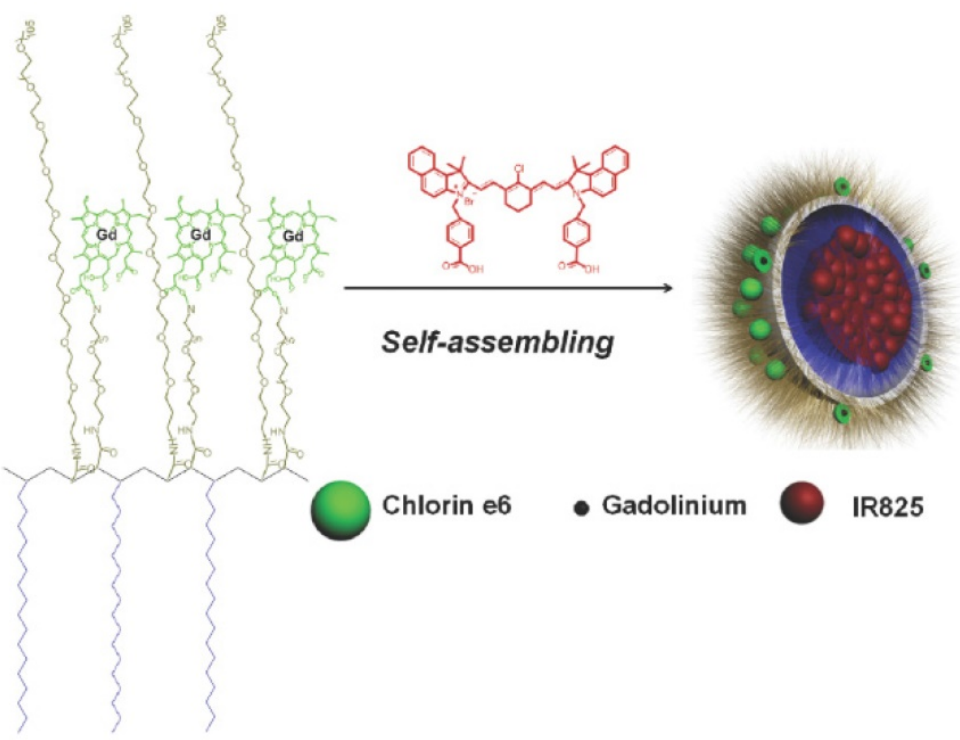

Figure 7. A scheme of illustrationof structure of $\mathbb{I R}_{825} @ \mathrm{C}_{18} \mathrm{PMH}-\mathrm{PEG}-\mathrm{Ce} 6-\mathrm{Gd}$ nanoprobe. Reproduced with permission from ref. 55 . Copyright $\odot 2014$ WILEY-VCH Verlag GmbH \& Co. KGaA, Weinheim.

Silicon is a widely used material to encapsulate imaging modalities because it has good light transmittance. In 2008, Brij M. Moudgil's group has designed a multimodal nanoprobe based on the encapsulating method [60]. Gadolinium doped gold particles as MRI contrast agents and gold nanoparticle as PA imaging contrast agents are encapsulated into the silicon shell, which possess no imaging modality.

\section{Conjugating different kinds of nanoparticles with covalent or non-covalent linkage}

The last method to construct "all in one" multimodal nanoprobe is referred to conjugate different functional components together. To distinguish a multimodal probe is designed by loading (encapsulating) or conjugating, there are two 
criterions for conjugating as following: i) chemical interaction (electronic interaction) between the imaging modality components; ii) the carrier must possess imaging modality. To illustrate the method, covalent and non-covalent examples will be shown in the following section.

Core-shell is a typical structure of multimodal nanoprobes [61-66]. Various kinds of methods have been developed to synthesize iron oxide core and gold shell structure [67-69]. Very recently, Chen Huabing's group have designed a novel method to construct magnetic gold nanoflowers (Figure 8) [70]. The as-prepared multimodal nanoprobe not only can be used as PA imaging and MRI contrast agent, but also holds surface-enhanced Raman spectroscopy ability. The iron oxide is prepared through co-precipitation method. To grow rough gold surface on the iron oxide nanoparticle, three reduction processes are carried out. The component of iron oxide is responsible for the MRI, while gold nanoparticles is used as PA imaging and surface-enhanced Raman spectroscopy contrast agent. More importantly, the rough surface of the whole nanoparticle plays an important role to enhance the surface-enhanced Raman spectroscopy signal because of the "sharp tip effect". As we can see, the multimodal nanoprobe satisfies both of the two rules. Covalent interaction is occurred between the components. Furthermore, the two components are contributed to the multifunctional imaging. In previous years, Xing Da's group has also developed a MRI and PA imaging multimodal nanoprobe based on iron oxide and gold nanostructure [71]. Gold core with organic shell multimodal nanoprobes have also designed. With the usage of the interaction between gold and dopamine, Liu gang's group has constructed a MRI and PA imaging nanoprobe [69]. Self-polymerization of the dopamine serve as PA imaging contrast agent because of their near-infrared absorption. Iron oxide is still used as the MRI contrast agent. Through this conjugating method, PA imaging and CT contrast agent has been constructed based on gold core with palladium shell. The reaction between carboxyl group and amine group is also used to construct this kind of multimodal nanoprobe. Gao et.al. have designed a nanoplatform based on the BSA-modified CuS nanoparticles utilizing the reaction amine groups of BSA. DTPA-Gd ${ }^{3+}$ ions with active carboxyl groups are conjugated on the surface of the nanoparticles to realize multimodal imaging [72].

The construction of multimodal nanoprobe can also be involved in non-covalent linkage. Cheng Zhen's group has designed a multimodal nanoprobe based on melanin nanoparticles (Figure 9) [73]. Functional metal ions such as iron ions $\left(\mathrm{Fe}^{3+}\right)$ and radioactive copper ions $\left({ }^{64} \mathrm{Cu}^{2+}\right)$ are then binding on the nanoplatform. Melanin is a natural pigment served as PA imaging contrast agents, while $\mathrm{Fe}^{3+}$ served as MRI contrast agents instead of gadolinium ions to make the nanoprobe more biocompatible. Radioactive ${ }^{64} \mathrm{Cu}^{2+}$ is used for positron emission tomography, which finally decay to zinc ions. All the imaging modality components are integrated through non-covalent interaction, which also can be metabolized in organism.
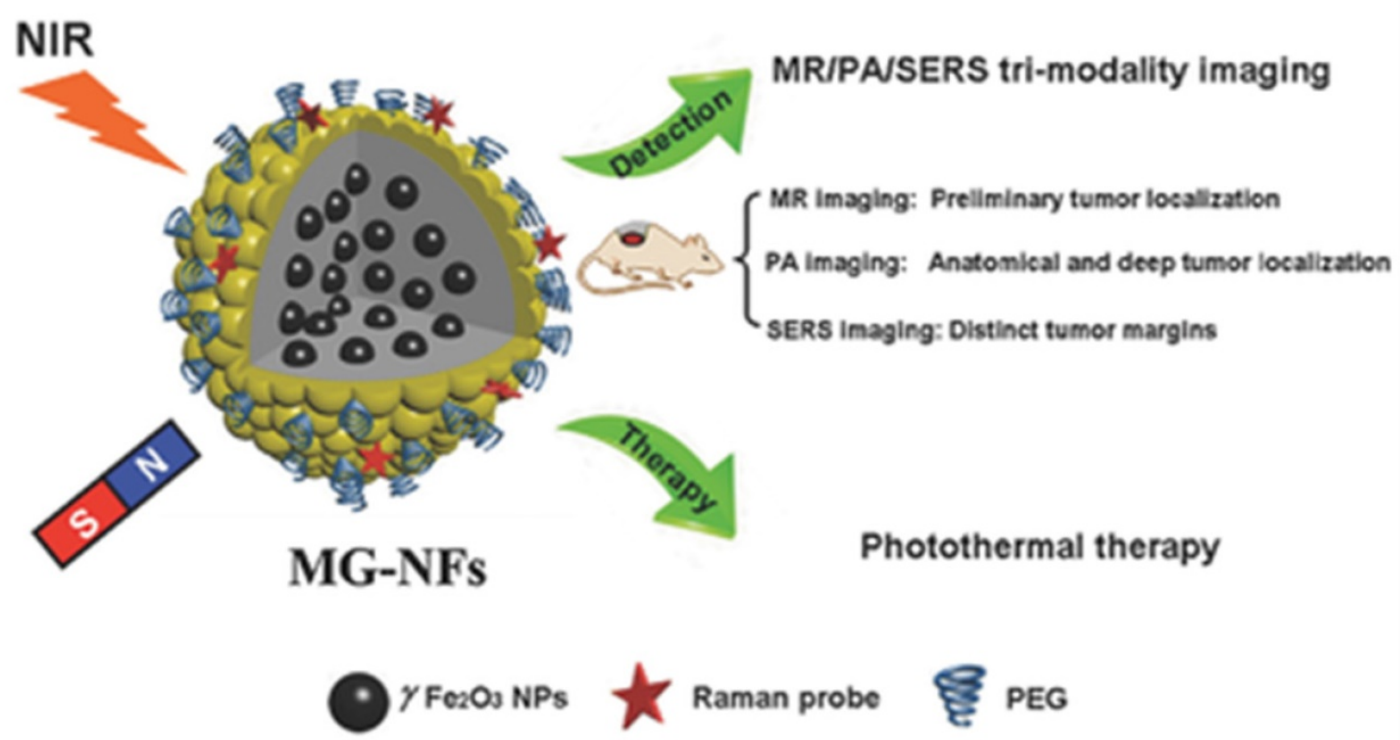

Figure 8. Illustration of core/shell $\mathrm{Fe}_{2} \mathrm{O}_{3} @$ @u magnetic gold nanoflowers for magnetic resonance/photoacoustic/SERS imaging and image-guided photothermal therapy of cancer. Reproduced with permission from ref. 70. Copyright @ 2015 WILEY-VCH Verlag GmbH \& Co. KGaA, Weinheim. 


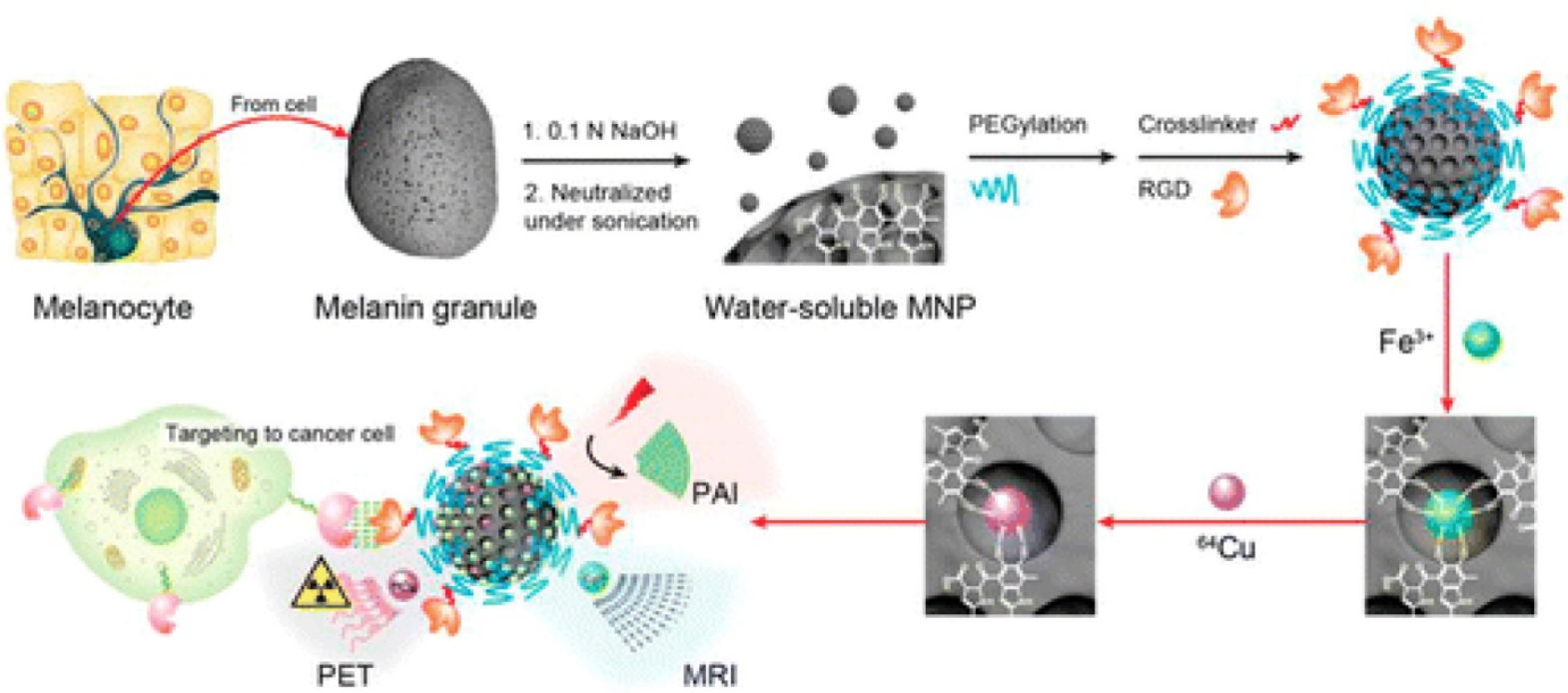

Figure 9. Multimodality molecular imaging of melanin nanoparticles. Reproduced from reference 73. (an open-access article by ACS author choice. This is an unofficial adaptation of an article that appeared in an ACS publication. ACS has not endorsed the content of this adaptation or the context of its use).

\section{PA-based multimodal biomedical imaging}

Various kinds of multimodal imaging nanoprobes are synthesized for biomedical imaging. They are tools that provide a chance to enhance the detection sensitivity, imaging contrast and spatial resolution. In this review, in vivo lymph node imaging and tumor imaging were considered as the two representative cases involved PA-based multimodal imaging based on multifunctional nanoprobes.

Abnormal lymph nodes are related to many diseases, especially at the early stage of cancer. Therefore, it is of great significance to image lymph node by the utilization of exogenously administered contrast enhancing agents. MRI, fluorescence imaging and positron emission tomography are involved in the lymph node imaging. In recent years, PA imaging as a noninvasive imaging technique is used for imaging lymph node. Multimodal nanoprobes based PA imaging have also been established. Zeng Wenbin's group has detected the lymph node by fluorescence imaging and PA imaging with the usage of multimodal nanoprobe which is synthesized by loading fluorescent dye into mesoporous silica nanoparticles (Figure 10) [74]. As mentioned before, a multimodal nanoprobe integrated MRI and PA imaging have also been used for lymph node imaging. The multimodal nanoprobe has been synthesized through doping method.

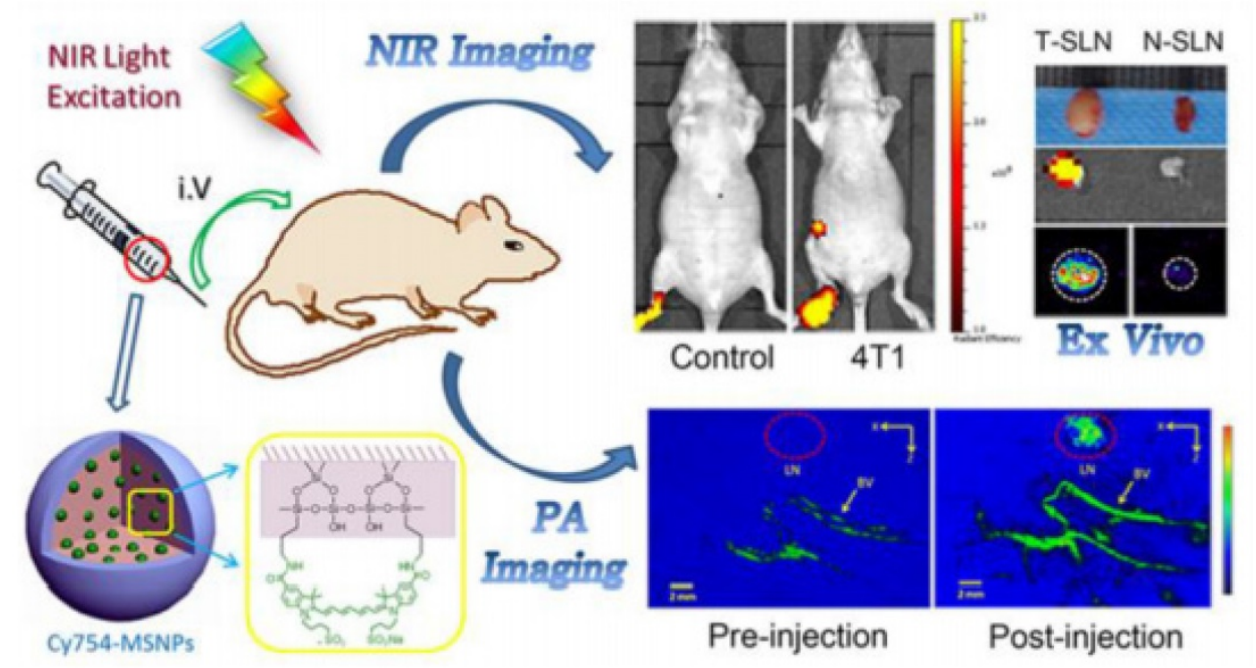

Figure 10. Schematic illustration of multimodal nanoprobes for lymph node imaging. Reproduced with permission from ref. 74. Copyright $\odot$ 2015, American Chemical Society. 


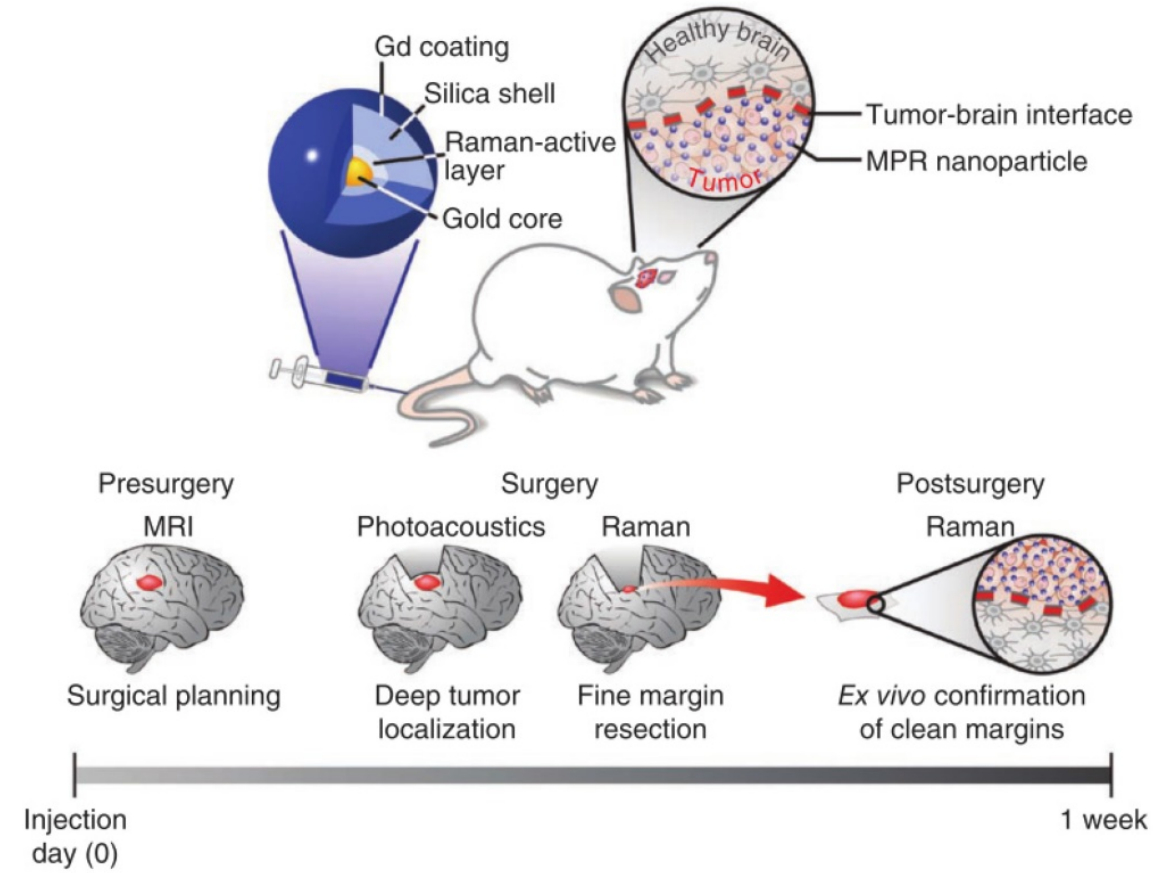

Figure 11. Triple-modality magnetic resonance/photoacoustic/SERS nanoprobes and image-guided surgery concept. Reproduced with permission from ref. 75. Copyright () 2012, Rights Managed by Nature Publishing Group.

Cancer remains a leading cause of morbidity and mortality. High-sensitivity multimodal imaging techniques for cancer early detection and image-guided therapeutics customized at the individual patient level are urgently needed. The developed multimodal molecular imaging methods based on novel contrast agents will have the advantages of guiding treatment and preventing tumor recurrence after treatment. Multimodal nanoprobes as a tool to enhance the sensitivity, imaging contrast and spatial resolution by integrating complementary merits of multiple imaging modalities have been used for tumor imaging and imaging-guide therapy. Sanjiv S Gambhir's group has constructed a multimodal nanoprobe combining PA imaging, MRI and Raman imaging (Figure 11) [75]. Gadolinium ions as MRI contrast agent, gold core responsible for PA imaging and trans-1, 2-bis (4-pyridyl)-ethylene as Raman molecular tag are loading into a silica shell. In this experiment, MRI can supply the tumor position in the whole brain, PA imaging provides three dimensional and high spatial resolution information of the brain tumor and Raman imaging offers the tumor margin because of its high sensitivity and high resolution. The multimodal nanoprobe combining the complementary merits of the imaging modalities exhibits excellent imaging ability for preoperative, intraoperative and post-surgery. Several multimodal nanoprobes that are constructed through other methods are applied for tumor imaging too.

\section{Summary and Perspectives for PA-based multimodal probes}

In this review, we have summarized the PA-based multimodal nanoprobes and their applications for biomedical imaging. We have classified the previous synthetic method of PA-based multimodal nanoprobes into two categories: "one for all" process and "all in one" process. We have simply introduced some common functional entities for the different synthetic methods. Furthermore, "all in one" process has been divided into three types including: i) doping different components into core material to realize multifunction; ii) encapsulating or loading different imaging components into one carrier; iii) conjugating different kinds of functional constituents with covalent or non-covalent linkage. To illustrate these processes clearly, each type is accompanies with examples by using the functional entities.

Since the review focus on the method to construct PA-based multimodal nanoprobes, we only choose nanoprobes combined PA imaging modality as examples. As a matter of fact, the methods are suitable for constructing other kinds of multimodal nanoprobes.

As can be seen from the examples, various multimodal nanoprobes have been prepared. 
However, there is still plenty of room to develop adequate multimodal nanoprobes. Nanoprobe integrated therapy function is a promising direction to design nanomaterials. For potential clinical translation, the components should be chosen carefully. Combining the materials approved by Food and Drug Administration maybe can accelerate the speed of clinical translation. Besides considering the components, surface modification, biodegradation, synthetic methods and targeting capability are should be taken into account.

The most promising method to construct multimodal nanoprobe is the concept of "one for all" and "doping functional components into core material". Less reaction steps make it much easier to control the construction of the nanoprobes. Just as an old saying in ancient China: the way to achieve great success an immortal deed tends to be the simplest one. In the future, simplest method should be developed to integrate multimodal imaging and therapy entities together.

\section{Acknowledgements}

The authors express many thanks to Dr. Zhang Xuanjun for critical reading and discussion. This work was supported by Grant from the University of Macau (Grant No. MYRG2014-00093-FHS, MYRG2015-00036-FHS, and MYRG2016-00110-FHS) and Grant from the Macao government (Grant No. FDCT 026/2014/A1 and FDCT 025/2015/A1).

\section{Competing Interests}

The authors have declared that no competing interest exists.

\section{References}

1. Zhang Y, Yang Y, Cai W. Multimodality Imaging of Integrin alpha(v)beta(3) Expression. Theranostics. 2011; 1: 135-148.

2. Kiessling F, Gaetjens J, Palmowski M. Application of Molecular Ultrasound for Imaging Integrin Expression. Theranostics. 2011; 1: 127-134.

3. Park JI, Jagadeesan D, Williams R, Oakden W, Chung S, Stanisz GJ, Kumacheva E. Microbubbles Loaded with Nanoparticles: A Route to Multiple Imaging Modalities. Acs Nano. 2010; 4: 6579-6586.

4. Stride E, Edirisinghe M. Novel microbubble preparation technologies. Soft Matter. 2008; 4: 2350-2359.

5. Kiessling F, Fokong S, Bzyl J, Lederle W, Palmowski M, Lammers T. Recent Advances in Molecular, Multimodal and Theranostic Ultrasound Imaging. Adv Drug Deliv Rev. 2014; 72: 15-27.

6. Cai X, Yang F, Gu N. Applications of magnetic microbubbles for theranostics. Theranostics. 2012; 2: 103-112.

7. Lusic H, Grinstaff MW. X-ray-computed tomography contrast agents. Chem Rev. 2012; 113: 1641-1666.

8. Lee N, Choi SH, Hyeon T. Nano-Sized CT Contrast Agents. Adv Mater. 2013; 25: $2641-2660$.

9. Lee DE, Koo H, Sun IC, Ryu JH, Kim K, Kwon IC. Multifunctional nanoparticles for multimodal imaging and theragnosis. Chem Soc Rev. 2012; 41: 2656-2672.

10. Liu Z, Kiessling F, Gaetjens J, Advanced Nanomaterials in Multimodal Imaging: Design, Functionalization, and Biomedical Applications. J Nanomater. 2010; 51:1-15.

11. Bell, AG. On the production and reproduction of sound by light. Am J Sci. 1880; 20: 305-324.

12. Nie L, Chen X. Structural and functional photoacoustic molecular tomography aided by emerging contrast agents. Chem Soc Rev. 2014; 43: 7132-7170.
13. Nune SK, Gunda P, Thallapally PK, Lin YY, Laird Forrest M, Berkland CJ. Nanoparticles for biomedical imaging. Expert Opin Drug Deliv. 2009; 6: 1175-1194.

14. Louie A. Multimodality imaging probes: design and challenges. Chem Rev. 2010; 110: 3146-3195.

15. Josephson L, Kircher MF, Mahmood U, Tang Y, Weissleder R. Near-infrared fluorescent nanoparticles as combined MR/optical imaging probes. Bioconjug Chem. 2002; 13: 554-560

16. Zhou M, Zhang R, Huang M, Lu W, Song S, Melancon MP, et al. A Chelator-Free Multifunctional Cu-64 CuS Nanoparticle Platform for Simultaneous Micro-PET/CT Imaging and Photothermal Ablation Therapy. J Am Chem Soc. 2010; 132: 15351-15358.

17. Dai Y, Xiao H, Liu J, Yuan Q, Ma PA, Yang D, et al. In Vivo Multimodality Imaging and Cancer Therapy by Near-Infrared Light-Triggered trans-Platinum Pro-Drug-Conjugated Upconverison Nanoparticles. J Am Chem Soc, 2013; 135: 18920-18929.

18. Guo W, Sun X, Jacobson O, Yan X, Min K, Srivatsan A, et al. Intrinsically Radioactive Cu-64 CuInS/ZnS Quantum Dots for PET and Optical Imaging: Improved Radiochemical Stability and Controllable Cerenkov Luminescence. Acs Nano. 2015; 9: 488-495.

19. Gao D, Zhang P, Sheng Z, Hu D, Gong P, Chen C, et al. Highly Bright and Compact Alloyed Quantum Rods with Near Infrared Emitting: a Potential Multifunctional Nanoplatform for Multimodal Imaging In Vivo. Adv Funct Mater. 2014; 24: 3897-3905.

20. Ma, M, Chen H, Shi J. Construction of smart inorganic nanoparticle-based ultrasound contrast agents and their biomedical applications. Science Bulletin. 2015; 60: 1170-1183.

21. $\mathrm{Hu} \mathrm{P}$, Liu $\mathrm{K}$, Deming $\mathrm{CP}$, Chen $\mathrm{S}$. Multifunctional graphene-based nanostructures for efficient electrocatalytic reduction of oxygen. J Chem Technol Biotechnol. 2015; 90: 2132-2151.

22. Cole JT, Holland NB. Multifunctional nanoparticles for use in theranostic applications. Drug Deliv Transl Re. 2015; 5: 295-309.

23. Baetke SC, Lammers T, Kiessling F. Applications of nanoparticles for diagnosis and therapy of cancer. Br J Radiol. 2015; 88: 20150207.

24. Hembury M, Chiappini C, Bertazzo S, Kalber TL, Drisko GL, Ogunlade O, et al. Gold-silica quantum rattles for multimodal imaging and therapy. PP Natl Acad Sci USA 2015; 112: 1959-1964.

25. Jin YD, Multifunctional Compact Hybrid Au Nanoshells: A New Generation of Nanoplasmonic Probes for Biosensing, Imaging, and Controlled Release. Acc Chem Res. 2014; 47:138-148.

26. Huang G, Yang S, Yuan Y, Xing D. Combining x-ray and photoacoustics for in vivo tumor imaging with gold nanorods. Appl Phys Lett. 2011; 99: 123701-123704

27. Mou J, Liu C, Li P, Chen $Y, X u H$, Wei C, et al. A facile synthesis of versatile $\mathrm{Cu} 2-\mathrm{xS}$ nanoprobe for enhanced MRI and infrared thermal/photoacoustic multimodal imaging. Biomaterials. 2015; 57: 12-21.

28. Poulose AC, Veeranarayanan S, Mohamed MS, Nagaoka Y, Aburto RR, Mitcham $\mathrm{T}$, et al. Multi-stimuli responsive $\mathrm{Cu} 2 \mathrm{~S}$ nanocrystals as trimodal imaging and synergistic chemo-photothermal therapy agents. Nanoscale. 2015; 7: 8378-8388.

29. Cheng L, Liu J, Gu X, Gong H, Shi X, Liu T, et al. PEGylated WS2 Nanosheets as a Multifunctional Theranostic Agent for in vivo Dual-Modal CT/Photoacoustic Imaging Guided Photothermal Therapy. Adv Mater. 2014; 26: $1886-1893$

30. Tan $\mathrm{C}$, Zhang $\mathrm{H}$. Wet-chemical synthesis and applications of non-layer structured two-dimensional nanomaterials. Nat Commun. 2015; 6: 7873-7886.

31. Tan C, Zhang H. Epitaxial Growth of Hetero-Nanostructures Based on Ultrathin Two-Dimensional Nanosheets. J Am Chem Soc. 2015; 137: 12162-12174.

32. Matthaei JF, DiMaio F, Richards JJ, Pozzo LD, Baker D, Baneyx F. Designing Two-Dimensional Protein Arrays through Fusion of Multimers and Interface Mutations. Nano Lett. 2015: 15. 5235-5239.

33. Manzeli S, Allain A, Ghadimi A, Kis A. Piezoresistivity and Strain-induced Band Gap Tuning in Atomically Thin MoS2. Nano Lett. 2015; 15: 5330-5335.

34. Dong $\mathrm{R}$, Pfeffermann $\mathrm{M}$, Liang $\mathrm{H}$, Zheng $\mathrm{Z}$, Zhu X, Zhang J, Feng X. Large-Area, Free-Standing, Two-Dimensional Supramolecular Polymer Single-Layer Sheets for Highly Efficient Electrocatalytic Hydrogen Evolution. Angew Chem Int Ed. 2015; 54: 12058-12063.

35. Dienel T, Kawai S, Söde H, Feng X, Muillen K, Ruffieux P, et al. Resolving Atomic Connectivity in Graphene Nanostructure Junctions. Nano Lett. 2015; 15: 5185-5190.

36. Song XR, Wang X, Yu SX, Cao J, Li SH, Li J, et al. Co9Se8 Nanoplates as a New Theranostic Platform for Photoacoustic/Magnetic Resonance Dual-Modal-Imaging-Guided Chemo-Photothermal Combination Therapy. Adv Mater. 2015; 27: 3285-3291.

37. Liu J, Wang C, Wang X, Wang X, Cheng L, Li Y, Liu Z. Mesoporous Silica Coated Single-Walled Carbon Nanotubes as a Multifunctional Light-Responsive Platform for Cancer Combination Therapy. Adv Funct Mater. 2015; 25: 384-392.

38. Liu Z, Liu J, Wang R, Du Y, Ren J, Qu X. An efficient nano-based theranostic system for multi-modal imaging-guided photothermal sterilization in gastrointestinal tract. Biomaterials. 2015; 56: 206-218.

39. Mou J, Li P, Liu C, Xu H, Song L, Wang J, et al. Ultrasmall Cu2-xS Nanodots for Highly Efficient Photoacoustic Imaging-Guided Photothermal Therapy. Small. 2015; 11: 2275-2283. 
40. Zha Z, Zhang S, Deng Z, Li Y, Li C, Dai Z. Enzyme-responsive copper sulphide nanoparticles for combined photoacoustic imaging, tumor-selective chemotherapy and photothermal therapy. Chem Comm. 2013; 49:3455-3457.

41. Liu X, Law WC, Jeon M, Wang X, Liu M, Kim C, et al. Cu2-xSe Nanocrystals with Localized Surface Plasmon Resonance as Sensitive Contrast Agents for In Vivo Photoacoustic Imaging: Demonstration of Sentinel Lymph Node Mapping. Adv Healthc Mater. 2013; 2: 952-957.

42. Ku G, Zhou M, Song S, Huang Q, Hazle J, Li C. Copper Sulfide Nanoparticles As a New Class of Photoacoustic Contrast Agent for Deep Tissue Imaging at $1064 \mathrm{~nm}$. Acs Nano. 2012; 6:7489-7496.

43. Ge J, Jia Q, Liu W, Guo L, Liu Q, Lan M, et al. Red-Emissive Carbon Dots for Fluorescent, Photoacoustic, and Thermal Theranostics in Living Mice. Adv Mater. 2015; 27: 4169-4177.

44. Wang S, Li X, Chen Y, Cai X, Yao H, Gao W, et al. A Facile One-Pot Synthesis of a Two-Dimensional MoS2/Bi2S3 Composite Theranostic Nanosystem for Multi-Modality Tumor Imaging and Therapy. Adv Mater. 2015; 27: 2775-2782.

45. Tian G, Zhang X, Zheng X, Yin W, Ruan L, Liu X, et al. Multifunctional Rb-X WO3 Nanorods for Simultaneous Combined Chemo-photothermal Therapy and Photoacoustic/CT Imaging. Small. 2014; 10: 4160-4170.

46. Gao D, Zhang P, Liu C, Chen C, Gao G, Wu Y, et al. Compact chelator-free Ni-integrated CuS nanoparticles with tunable near-infrared absorption and enhanced relaxivity for in vivo dual-modal photoacoustic/MR imaging. Nanoscale. 2015; 7: 17631-17636.

47. Zhu W, Liu K, Sun X, Wang X, Li Y, Cheng L, Liu Z., et al. Mn2+-Doped Prussian Blue Nanocubes for Bimodal Imaging and Photothermal Therapy with Enhanced Performance. ACS Appl Mat Interfaces 2015; 7: 11575-11582.

48. Song J, Yang X, Jacobson O, Lin L, Huang P, Niu G, et al. Sequential Drug Release and Enhanced Photothermal and Photoacoustic Effect of Hybrid Reduced Graphene Oxide-Loaded Ultrasmall Gold Nanorod Vesicles for Cancer Therapy. Acs Nano. 2015; 9: 9199-9209.

49. Zhang Y, Jeon M, Rich LJ, Hong H, Geng J, Zhang Y, et al. Non-invasive multimodal functional imaging of the intestine with frozen micellar naphthalocyanines. Nat Nanotech. 2014; 9: 631-638

50. Wang $\mathrm{YH}$, Chen SP, Liao AH, Yang YC, Lee CR, Wu CH, et al. Synergistic delivery of gold nanorods using multifunctional microbubbles for enhanced plasmonic photothermal therapy. Sci Rep. 2014; 4: 5685-5693.

51. Ray A, Mukundan A, Xie Z, Karamchand L, Wang X, Kopelman R. Highly stable polymer coated nano-clustered silver plates: a multimodal optical contrast agent for biomedical imaging. Nanotechnology. 2014; 25: 445104-445113.

52. Nie LM, Huang P, Li W, Yan X, Jin A, Wang Z, et al. Early-Stage Imaging of Nanocarrier-Enhanced Chemotherapy Response in Living Subjects by Scalable Photoacoustic Microscopy. Acs Nano. 2014; 8: 12141-12150.

53. Huynh E, Jin CS, Wilson BC, Zheng G. et al. Aggregate Enhanced Trimodal Porphyrin Shell Microbubbles for Ultrasound, Photoacoustic, and Fluorescence Imaging. Bioconjug Chem. 2014; 25: 796-801.

54. Guo M, Mao H, Li Y, Zhu A, He H, Yang H, et al. Dual imaging-guided photothermal/photodynamic therapy using micelles. Biomaterials. 2014; 35: 4656-4666.

55. Gong H, Dong Z, Liu Y, Yin S, Cheng L, Xi W, et al. Engineering of Multifunctional Nano-Micelles for Combined Photothermal and Photodynamic Therapy Under the Guidance of Multimodal Imaging. Adv Funct Mater. 2014; 24: 6492-6502.

56. Yang K, Hu L, Ma X, Ye S, Cheng L, Shi X, et al. Multimodal Imaging Guided Photothermal Therapy using Functionalized Graphene Nanosheets Anchored with Magnetic Nanoparticles. Adv Mater. 2012; 24: 1868-1872.

57. Yu J, Yin W, Zheng X, Tian G, Zhang X, Bao T, et al. Smart MoS2/Fe3O4 Nanotheranostic for Magnetically Targeted Photothermal Therapy Guided by Magnetic Resonance/Photoacoustic Imaging. Theranostics, 2015; 5: 931-945.

58. Yan X, Hu H, Lin J, Jin AJ, Niu G, Zhang S, et al. Optical and photoacoustic dual-modality imaging guided synergistic photodynamic/photothermal therapies. Nanoscale. 2015; 7: 2520-2526.

59. Lin J, Wang S, Huang P, Wang Z, Chen S, Niu G, et al. Photosensitizer-Loaded Gold Vesicles with Strong Plasmonic Coupling Effect for Imaging-Guided Photothermal/Photodynamic Therapy. Acs Nano. 2013; 7: 5320-5329.

60. Sharma P, Brown SC, Bengtsson N, Zhang Q, Walter GA, Grobmyer SR, et al. Gold-Speckled Multimodal Nanoparticles for Noninvasive Bioimaging. Chem Mater. 2008; 20: 6087-6094.

61. Yang M, Fan Q, Zhang R, Cheng K, Yan J, Pan D, et al. Dragon fruit-like biocage as an iron trapping nanoplatform for high efficiency targeted cancer multimodality imaging. Biomaterials. 2015; 69: 30-37.

62. Yu J, Yang C, Li J, Ding Y, Zhang L, Yousaf MZ, et al. Multifunctional Fe5C2 Nanoparticles: A Targeted Theranostic Platform for Magnetic Resonance Imaging and Photoacoustic Tomography-Guided Photothermal Therapy. Adv Mater. 2014; 26: 4114-4120.

63. Chen M, Tang S, Guo Z, Wang X, Mo S, Huang X, et al. Core-Shell Pd@Au Nanoplates as Theranostic Agents for In-Vivo Photoacoustic Imaging, CT Imaging, and Photothermal Therapy. Adv Mater. 2014; 26: 8210-8216.

64. Wang C, Ma X, Ye S, Cheng L, Yang K, Guo L, et al. Protamine Functionalized Single-Walled Carbon Nanotubes for Stem Cell Labeling and In Vivo Raman/Magnetic Resonance/Photoacoustic Triple-Modal Imaging. Adv Funct Mater. 2012; 22: 2363-2375.

65. Jin Y, Jia C, Huang SW, O'Donnell M, Gao X. Multifunctional nanoparticles as coupled contrast agents. Nat Commun. 2010; 1: 41-49.
66. Kim JW, Galanzha EI, Shashkov EV, Moon HM, Zharov VP. Golden carbon nanotubes as multimodal photoacoustic and photothermal high-contrast molecular agents. Nat Nanotech. 2009; 4: 688-694.

67. Bai LY, Yang XQ, An J, Zhang L, Zhao K, Qin MY, et al. Multifunctional magnetic-hollow gold nanospheres for bimodal cancer cell imaging and photothermal therapy. Nanotechnology. 2015; 26: 315701-315713.

68. Wu CH, Cook J, Emelianov S, Sokolov K. Multimodal Magneto-Plasmonic Nanoclusters for Biomedical Applications. Adv Funct Mater. 2014; 24: 6862-6871

69. Lin LS, Cong ZX, Cao JB, Ke KM, Peng OL, Gao J, et al. Multifunctional Fe3O4@Polydopamine Core-Shell Nanocomposites for Intracellular mRNA Detection and Imaging-Guided Photothermal Therapy. Acs Nano. 2014; 8: 3876-3883.

70. Huang J, Guo M, Ke H, Zong C, Ren B, Liu G, et al. Rational Design and Synthesis gamma Fe2O3@Au Magnetic Gold Nanoflowers for Efficient Cancer Theranostics. Adv Mater. 2015; 27: 5049-5056.

71. Zhou T, Wu BY, Xing D. Bio-modified Fe3O4 core/Au shell nanoparticles for targeting and multimodal imaging of cancer cells. J Mater Chem. 2012; 22: $470-477$

72. Gao D, Sheng Z, Liu Y, et al. Protein-Modified CuS Nanotriangles: A Potential Multimodal Nanoplatform for In Vivo Tumor Photoacoustic/Magnetic Resonance Dual-Modal Imaging. Adv Healthc Mater, 2017; doi.10.1002/adhm.201601094.

73. Fan Q, Cheng K, Hu X, Ma X, Zhang R, Yang M, et al. Transferring Biomarker into Molecular Probe: Melanin Nanoparticle as a Naturally Active Platform for Multimodality Imaging. J Am Chem Soc, 2014; 136: 15185-15194.

74. Liu Z, Rong P, Yu L, Zhang X, Yang C, Guo F, et al. Dual-Modality Noninvasive Mapping of Sentinel Lymph Node by Photoacoustic and Near-Infrared Fluorescent Imaging Using Dye-Loaded Mesoporous Silica Nanoparticles. Mol Pharm. 2015; 12: 3119-3128.

75. Kircher MF, De La Zerda A, Jokerst JV, Zavaleta CL, Kempen PJ, Mittra E, et al. A brain tumor molecular imaging strategy using a new triple-modality MRI-photoacoustic-Raman nanoparticle. Nat Med. 2012; 18: 829-834. 